\title{
Intestinal spirochaetosis
}

\author{
T.C.N. Lo, R.C. Heading and H.M. Gilmour ${ }^{1}$ \\ Department of Medicine and ${ }^{1}$ Department of Histopathology, University of Edinburgh, Edinburgh, UK
}

\begin{abstract}
Summary: Two cases of intestinal spirochaetosis are described. The first case improved with treatment while the second case improved spontaneously without any intervention. Controversy over treatment and pathogenicity of intestinal spirochaetosis is discussed with review of previous publications.
\end{abstract}

\section{Introduction}

Infection of the human gastrointestinal tract by spirochaetes has been recognized for a long time, though their pathogenicity remains unclear. Spirochaetes have been found in the faeces of apparently healthy individuals with Fantham $^{1}$ reporting an incidence of $1.2 \%$ among British soldiers. This incidence increased to $30 \%$ in Chicago $^{2}$ and approached $100 \%$ in West Africa $^{3}$ illustrating wide geographical variation. In hospital patients undergoing rectal biopsy, the prevalence of 'rectal spirochaetosis' was found to range from $1.9 \%{ }^{4}$ to $6.9 \% .^{5}$ Increased incidence has also been reported in homosexual men with a history of anal intercourse ${ }^{6}$ but the relationship of these spirochaetes to intestinal disease remains unclear due to co-existence of other known pathogens. Some reports have suggested that 'intestinal spirochaetosis', the overgrowth of these organisms in rectum and colon, is associated with various abdominal symptoms including pain, diarrhoea and rectal bleeding ${ }^{7,8}$ which will respond to treatment. ${ }^{9,10}$

We report two cases of intestinal spirochaetosis which illustrate that heavy colonic involvement can occur with minimal involvement and even sparing of the rectum, and that treatment is indicated with prolonged symptoms.

\section{Case report}

\section{Case 1}

A 19 year old girl presented with a 3 month history of lower abdominal discomfort, watery diarrhoea with variable frequency up to six times per day, and occasional passage of small amounts of mucus and blood per rectum. Her symptoms started 3 weeks

Correspondence: T.C.N. Lo, M.R.C.P., Department of Integrated Medicine, Leicester General Hospital, Gwendolen Road, Leicester LE5 4PW, UK.

Accepted: 1 July 1993 after her return from a camping holiday in Italy. Physical examination was unremarkable. Microscopy and microbiological examination of the stool revealed no abnormality. Other investigations including jejunal biopsy and flexible sigmoidoscopy with multiple biopsies of the distal descending colon and rectum were all normal. Owing to her reluctance for further endoscopic examination, a colonoscopy was only performed 8 months later when her symptoms persisted. There was no macroscopic abnormality identified at colonoscopy, but a heavy infection of spirochaetes was seen under light microscopy from all the biopsies obtained from her proximal colon and caecum (Figure 1). Spirochaetes were not seen in biopsies obtained from her distal descending colon, sigmoid colon and rectum.

In view of the uncertainty over the significance of this finding, she was just followed up for another 6 months during which time her symptoms persisted. She was subsequently started on a 4 week course of metronidazole $(200 \mathrm{mg}$ three times a day). Her symptoms resolved completely within 2 weeks of treatment. Repeat colonoscopy with biopsies 2 months later revealed absence of spirochaetes in any colorectal biopsy. She has since remained asymptomatic for 3 years.

\section{Case 2}

A 18 year old male student presented with a 5 week history of loose bowel motions up to four times per day with occasional streaky fresh rectal bleeding. $\mathrm{He}$ had no recent foreign travel and denied homosexual practice. Physical examination was unremarkable. Investigations including stool microscopy and microbiological examination, rigid sigmoidoscopy and barium enema were all normal. However, rectal biopsy revealed mild mucosal oedema with presence of patchy spirochaete infection (Figure 2). No active inflammation was present and the gland architecture appeared normal with no reduction in globet cells. Subsequent 


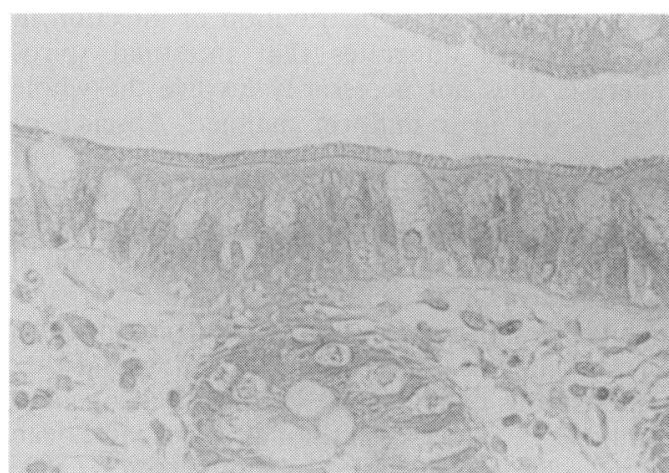

Figure 1 Case 1: colonic biopsy with spirochaetes on luminal aspect of surface columnar cells (haematoxylin and eosin, $\times 160$ ).

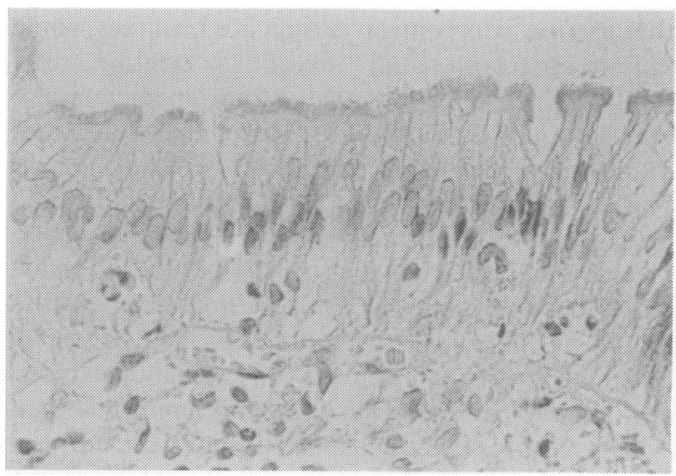

Figure 2 Case 2: rectal biopsy showing surface spirochaetes (haematoxylin and eosin, $\times 160$ ).

colonoscopy with multiple biopsies showed extensive spirochaetes infection throughout the whole large bowel from rectum to caecum. Attempts to culture these organisms according to the method described by Tompkins et al. ${ }^{11}$ failed. No medical treatment was offered because his symptoms which were only mild initially, appeared to be improving spontaneously. His symptoms resolved completely within 8 months and repeat colonoscopy with biopsies showed an absence of spirochaetes. He remains asymptomatic after 20 months.

\section{Discussion}

Spirochaetal infection has been identified in biopsies obtained from rectum and colon, as well as in appendicectomy specimens. ${ }^{12}$ Under light micro- scopy stained by haematoxylin and eosin (H\&E), intestinal spirochaetosis is characterized by the presence of a fuzzy blue (haematophilic) line at the brush border of the mucosal surface. With electron microscopy this line is found to consist of numerous spiral micro-organisms situated between and parallel to the microvilli. They appear to attach end on and orient at right angles to the luminal cell membrane but not penetrate them ${ }^{5,13}$ (Figure 3).

The taxonomy of these organisms is still in debate and it remains unclear which type of spirochaete infects human bowel. Originally, they were called Spirochaete eurygyrata. ${ }^{1,14}$ Based on their readiness to be stained by H\&E and periodic acid-Schiff, Lee $e t$ al. ${ }^{5}$ suggested that they should be classified with Borrelia rather than the Treponemata. Later, Hovind-Hougen et al. ${ }^{15}$ named these organisms Brachyspira aalborgi and proposed that they should constitute a new genus. Close phenotypic similarity between the human isolates and the non-pathogenic porcine intestine spirochaetes was demonstrated by Tompkins et al. ${ }^{11}$ using anaerobic culture of blood agar incorporated with spectinomycin, but both of them appeared to differ from Brachyspira aalborgi. It

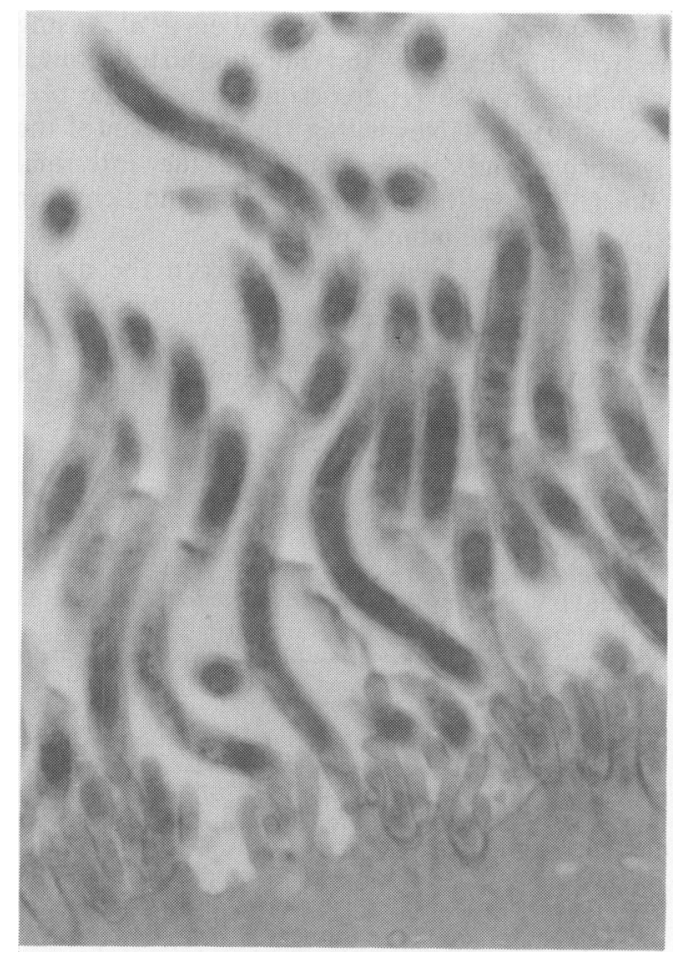

Figure 3 Case 1: transmission electron micrograph of spirochaetes showing interdigitation of organisms between surface microvilli $(\times 25,000)$. 
seems, therefore, human intestinal spirochaetes are heterogeneous and further refinements in classification are required.

The clinical importance of intestinal spirochaetosis in human remains controversal. Takeuchi et $a l .{ }^{4}$ and Gear and Dobbins ${ }^{16}$ considered the spirochaetes to be commensals and part of normal enteric flora. The lack of association between specific gastrointestinal symptoms and absence of mucosal inflammation despite microscopic presence of spirochaetes convinced both Lee et al..$^{5}$ and Nielson et al..$^{13}$ that colorectal spirochaetosis is without clinical significance.

However, Gad et al. ${ }^{17}$ reported four cases of longstanding diarrhoea in which intestinal spirochaetosis was the only pathologic finding and diarrhoea disappeared after treatment with neomycin. Douglas and Crucioli ${ }^{8}$ attributed two cases of acute diarrhoea and rectal bleeding to intestinal spirochaetosis because both cases improved after treatment with metronidazole. They proposed that, being a treatable cause, spirochaetes should be looked for in all rectal biopsies. Cotton et al. ${ }^{18}$ also suggested that spirochaetosis should be borne in mind when any homosexual patients present with unexplained diarrhoea and rectal bleeding.

Further supports to the invasive tendency and pathogenicity of spirochaetes were offered by Antonakopoulos et al. ${ }^{19}$ and Gebbers et al ${ }^{10}$ using electron microscopic and immunohistochemical techniques. These groups demonstrated the presence of spirochaetes within the cytoplasm of the absorptive and globlet cells of the intestinal mucosa, sub-epithelial macrophages and Schwann cells deep in the lamina propria.

The temporal relationship between the disappearance of spirochaetes and symptomatic improvement in both patients reported here gives circumstantial support to a pathological role for

\section{References}

1. Fantham, H.B. Observations on Spirochaeta eurygyrata as found in human faeces. $\mathrm{Br}$ Med J 1916, 1: 815-816.

2. Parr, L.W. Intestinal spirochaetes. J Infect Dis 1923, 33: 369-383.

3. Macfie, J.W.S. The prevalence of Spirochaeta euygyrata in Europeans and natives in the Golden Coast. Lancet 1919, 1: $336-340$.

4. Takeuchi, A., Jervis, H.R., Nakazaira, H. et al. Spiral shaped organisms on the surface colonic epithelium of the monkey and man. Am J Clin Nutr 1974, 27: 1287-1296.

5. Lee, F.D., Kraszewski, A., Gordon, J. et al. Intestinal spirochaetosis. Gut 1971, 12: 126-133.

6. McMillan, A. \& Lee, F.D. Sigmoidoscopic and microscopic appearance of rectal mucosa in homosexual men. Gut 1981, 22: $1035-1041$.

7. Lee, F.D., Krasezewski, A., Gordon, J. et al. Intestinal spirochaetosis. Br Med J 1967, 3: 718-719.

8. Douglas, J.G. \& Crucioli, V. Spirochaetosis: a remediable cause of diarrhoea and rectal bleeding. Br Med J 1981, 283: 1362. these organisms. The distribution of infection in our first case illustrates that intestinal spirochaetosis does not necessarily involve the whole large bowel in a uniform manner. Absence of spirochaetes on rectal biopsy does not rule out more proximal infection. Hence, it is potentially misleading to consider infection of rectum, 'rectal spirochaetosis', to be synonymous with 'intestinal spirochaetosis' which implies much more extensive involvement. In retrospect, we should have perhaps treated our first case earlier; but our second case demonstrates convincingly that spontaneous and complete clearance of intestinal spirochaetes can occur without any medical intervention. The difference in the outcome of these two cases cannot be explained by the extent of infection because microscopic findings clearly showed that our second patient had a much more extensive involvement.

Review of the literature failed to identify any objective criteria which offers guidance on when therapeutic intervention is indicated. In fact, some major gastrointestinal textbooks contain only minimal information on intestinal spirochaetosis and have not included it as one of the differential diagnosis of diarrhoea and rectal bleeding.

In conclusion, our two cases offer support that human intestinal spirochaetes are enteropathic and hence intestinal spirochaetosis should be excluded when dealing with patients with diarrhoea and rectal bleeding. Furthermore, absence of spirochaetes on rectal biopsy only excludes rectal spirochaetosis but not intestinal spirochaetosis which requires examination with multiple biopsies by flexible sigmoidoscopy and/or colonoscopy. Lastly, treatment of intestinal spirochaetosis should be guided by the presence of persistent symptoms and not by the extent of colorectal infection.

9. Burns, D.G. \& Hayes, M.M. Rectal spirochaetosis: symptomatic response to metronidazole and mebendazole. $S \mathrm{Afr}$ Med J 1985, 68: 335-336.

10. Gebbers, J.O., Ferguson, D.J.P., Mason, C. et al. Spirochaetosis of the human rectum with an intraepithelial mast cell and IgE plasma cell response. Gut 1987, 28: 588- 593 .

11. Tompkins, D.S., Foulkes, S.J., Godwin, P.G.P. et al. Isolation and characterization of intestinal spirochaetes. $J$ Clin Pathol 1986, 39: 535-541.

12. Henrik-Nielson, R., Lundbeck, F.A., Stubb-Teglbjaerg, P. et al. Intestinal spirochaetosis of the vermiform appendix. Gastroenterology 1985, 88: 971-977.

13. Nielson, R.H., Orholm, M., Pederson, J.O. et al. Colorectal spirochaetosis: clinical significance of the infestation. Gastroenterology 1983, 85: 62-67.

14. Hogue, M.J. Spirochaete eurygyrata. J Exp Med 1922, 36: 617-626. 
15. Hovind-Hougen, K., Birch-Anderson, A., Nielson, R.H. et al. Intestinal spirochaetosis: morphological characterization and cultivation of Spirochaete Brachyspira aalborgi. J Clin Microbiol 1982, 16: 1127-1136.

16. Gear, E.V.\& Dobbins, W.O. Rectal biopsy. Gastroenterology 1968, 55: 522-544.

17. Gad, A., Willen, R., Furugard, K. et al. Intestinal spirochaetosis as a cause of longstanding diarrhoea. Upsala $J$ Med Sci 1977, 82: 49-54.
18. Cotton, D.W.K., Kirkham, N. \& Hicks, D.A. Rectal spirochaetosis. Br J Vener Dis 1984, 60: 106-109.

19. Antonakopoulos, G., Newman, J. \& Wilkinson, M. Intestinal spirochaetosis: an electron microscopic study of an unusual case. Histopathology 1982, 6: 477-488. 